Rev. salud pública. 14 (2): 325-339, 2012

\title{
Aproximación a la complejidad de las zoonosis en Colombia
}

\section{A complexity-based approach to zoonoses in Colombia}

\author{
Angela N. Agudelo-Suárez \\ Clínica Veterinaria Zooricatos. Pereira, Colombia. naportvet@hotmail,com \\ Recibido 6 Enero 2012/Enviado para Modificación 6 Marzo 2012/Aceptado 30 Abril 2012
}

\section{RESUMEN}

Para contribuir al propósito de mejorar la integralidad y eficacia de las políticas y programas de control, la presente reflexión intenta responder la siguiente pregunta: ¿Cómo aproximarse a la comprensión de las zoonosis en Colombia desde enfoques de complejidad? El ensayo está conformado por tres partes principales. En la primera se examinan las zoonosis como problema complejo y la reiterada tendencia de las políticas y programas de control de afrontarlas como un problema simple, con respuestas de origen unidisciplinario y reduccionistas. En la segunda parte se sintetiza el estatus actual de la complejidad como paradigma y las ventajas que tendría la utilización del mismo en el análisis, la formulación de políticas y de intervenciones. En la tercera parte se plantean los posibles campos de trabajo que sería necesario desarrollar para abordar las zoonosis en Colombia desde las ciencias de la complejidad.

Palabras Clave: Zoonosis, complejidad, sistemas complejos, Colombia (fuente: DeCS, BIREME).

\begin{abstract}
The present reflection was aimed at making a contribution towards improving control policy and programs integrality and efficacy in an attempt to answer the following question, "How can understanding zoonoses in Colombia be approached from a complexity-based approach?" The first part of this article examines zoonoses as a complex problem and the reiterated tendency of control policy and programs to deal with them as a simple problem, involving unidisciplinary and reductionist responses. The current status of complexity is synthesized as a paradigm, as are the advantages of using it in analysis and formulating policy and interventions. Possible fields of work are proposed for development when approaching zoonoses in Colombia from a complexity science viewpoint.
\end{abstract}

Key Words: Zoonoses, complexity, complex system, Colombia (source: MeSH, NLM). 
$\mathrm{E}$ n el año de 1959 la OMS definió las zoonosis como “... las enfermedades e infecciones que se transmiten de los animales al hombre y visceversa". Esta definición básica se conserva, a pesar de múltiples propuestas que apuntan a detallarla o adicionarle conceptos (1).

Para clasificar las zoonosis se han utilizado como criterios centrales, más no únicos, el agente infeccioso que la produce (virales, parasitarias, bacterianas, etc.) y el modo de transmisión (1-3). Así, por ejemplo, se consideran las zoonosis directas (se transmiten en una sola especie vertebrada, como la rabia), las ciclo-zoonosis (requieren más de una especia vertebrada, como las teniosis), las meta-zoonosis (requieren vertebrado e invertebrados, como la malaria y el Dengue) y las zapro-zoonosis (reservorios inanimados y hospederos vertebrados, como la histoplasmosis).

\section{Importancia de las zoonosis}

En el siglo pasado y en el presente, las zoonosis se han convertido en un problema de importancia mundial, a pesar de que gran parte de la morbilidad y mortalidad se concentran en los países en desarrollo y pobres. Aunque en muchos países se ha logrado controlar algunas de las zoonosis, en el panorama mundial de salud pública la importancia de las zoonosis se debe a su persistencia, carácter emergente y reemergente (4).

Al menos tres aspectos han contribuido a este resultado: por una parte, las tendencias estructurales recientes que han dado lugar a la globalización, las transiciones epidemiológicas de los países y el cambio climático, inciden con fuerza en la dinámica de las enfermedades infecciosas; por otra parte, el alcance y los tipos de respuestas políticas, que pese a contar con marcos analíticos amplios, suelen traducirse en decisiones programáticas e institucionales reduccionistas. Esto contrasta con la creciente conciencia sobre la relación entre zoonosis y múltiples factores sociales, económicos, ambientales y similares (2); en tercer lugar, el cuerpo principal de la base cognitiva de las decisiones, políticas y programas, es de tipo unidisciplinario.

En la fase actual de globalización se han consolidado los fenómenos de transición epidemiológica y de cambio climático. Todos los países han presentado, desde hace muchos años, cambios importantes en su perfil epidemiológico, como resultado de las transformaciones sociales, económicas y culturales. La transición epidemiológica ha llevado a reem- 
plazar las enfermedades transmisibles por las enfermedades crónicas, como causas principales de morbilidad y mortalidad (5). A esto se han agregado los efectos progresivos del cambio climático que inducen polución de organismos patógenos y el desbalance entre salud humana, animal y ambiental (6). Quizá por estas razones el $60 \%$ de las enfermedades transmisibles han llegado a corresponder a las zoonosis, de las cuales el $72 \%$ provienen de la fauna silvestre $(5,7-9)$. En el marco de estas tendencias, globalmente dominantes, las enfermedades transmisibles y las zoonosis siguen siendo entidades de primer orden en salud pública de muchos países.

Por décadas, la Organización Mundial de la Salud (OMS) y la Organización Panamericana de la Salud (OPS) han formulado directrices y recomendaciones para orientar a los países en el control de las zoonosis. Estas formulaciones consideran los múltiples factores, generales y específicos, que inciden en las zoonosis. A esto debe agregarse el enfoque de Determinantes Sociales de la Salud, que introdujo algunos criterios de jerarquización y ordenamiento de factores que hacen parte de las cadenas causales de las enfermedades (10).

Desde estos marcos analíticos amplios, cada país requiere precisar sus políticas con base en los determinantes específicos, en lo que tiene que ver con las zoonosis. La experiencia muestra que en muchos países el control de las zoonosis ha pasado por enfoques y políticas reduccionistas (desconocimiento de las interacciones de los factores causales), unilaterales (prioridades de acción excluyentes) y discontinuas o circunstanciales (sólo cuando se presentan brotes epidémicos y epizootias). Adicionalmente, muchos países no cuentan con recursos suficientes para implementar programas de control eficaces, continuos y duraderos.

\section{Políticas y conocimiento}

Cabe destacar que la base cognitiva de las decisiones, políticas y programas, de tipo unidisciplinario, ha sido provista por la epidemiología, enfocada en los factores de riesgo, en un esquema que subordina, convirtiéndolas en meros complementos, corrientes de pensamiento pertenecientes a disciplinas como la economía y la sociología. Se trata de aproximaciones basadas en saberes fragmentarios, unilaterales, débilmente multicausales, inmersas en esquemas de desarrollo multidisciplinarios o multiprofesionales limitados. Sin embargo, debe reconocerse que estos enfoques han logrado algunos éxitos notables, más ocasionales que continuos, pero esto no debe ocultar 
las insuficiencias manifiestas de las políticas y programas de control de las zoonosis. Por tanto, debe reafirmarse que el conocimiento proporcionado por disciplinas que consideran la problemática de las zoonosis de manera reduccionista, puede conducir a decisiones y políticas ineficaces, lo cual ha sido reconocido desde décadas atrás (11). Así mismo, sin desconocer los demás factores que han contribuido a la situación actual de las zoonosis, es necesario ubicar como de importancia clave, el efecto del tipo de conocimiento sobre las zoonosis con respecto a las decisiones y políticas que deben apuntar a las respuestas de fondo y estratégicas.

\section{Problema complejo}

Para superar el circuito que pone en relación asimétrica potentes fuerzas sociales, políticas y ambientales, operantes en diferentes ámbitos de la sociedad, muchos de los cuales estimulan las zoonosis, frente a políticas reduccionistas, débiles y unilaterales, destinadas a controlarlas, es indispensable retomar las zoonosis como un problema complejo, aún sin conceptualizar las mismas como un sistema complejo.

Las caracterizaciones más elementales de las zoonosis como un problema complejo provienen de algunas corrientes de pensamiento de las ciencias sociales en su ejercicio autónomo frente a su objeto de estudio. En general, gran parte de las ciencias sociales consideran las sociedades como problemas complejos, por definición, en especial por la diferenciación y especialización internas debido al desarrollo del proceso productivo tecnológico y la división del trabajo. Y las zoonosis se encuentran en medio de estructuras sociales, como se desprende, para mencionar solo algunos, de los aportes de la economía agraria, la sociología estructuralista (12), la historia, la ciencia política y otras. Los estudios de los sistemas productivos agropecuarios y su relación con el mercado, el desenvolvimiento de las estructuras sociales, la historia del poblamiento, de la frontera agrícola interior, las colonizaciones y migraciones, las dinámicas sociales de los cultivos ilícitos, los conflictos armados, entre muchos otros, sugieren potentes relaciones no lineales e interacciones entre los agentes de las zoonosis (seres humanos, animales, agentes infecciosos y vectores) que, a su vez, pasan por las interacciones de los componentes de la estructura social y esta opera como un todo.

En Colombia las zoonosis hacen parte de su identidad e historia y comparten las características de persistencia descritas o se comportan 
como fenómenos emergentes y reemergentes. En este sentido, tenemos ejemplos como la Rabia, la Leishmaniasis, la Leptospirosis, la Encefalitis Equina Venezolana y la Brucelosis $(8,13-20)$.

De la misma manera que se ha planteado atrás y por las mismas razones, uno de los caminos para avanzar en el control de las zoonosis en Colombia, requiere reenfocar las mismas como un problema complejo. Pero esto, por sí solo, no es suficiente, ya que desde un modelo complejo se puede retornar con facilidad a respuestas reduccionistas, con sus prácticas multiprofesionales limitadas. En otras palabras, un problema complejo supone una determinación compleja, la cual usualmente es evasiva para los enfoques epidemiológicos, que aún desde perspectivas multicausales, arriban con facilidad en el reduccionismo interpretativo. Esto no es un problema voluntarista o de "miopía metodológica", sino resultado de la vocación ontológica y epistemológica de la epidemiología. Por estas razones se cree necesario y conveniente adoptar los enfoques de las ciencias de la complejidad y apuntar a esquemas más amplios, interdisciplinarios y transdisciplinarios. De esta manera, el abordaje analítico de la problemática de las zoonosis en Colombia, como un aporte para mejorar la calidad, alcance y eficacia de las políticas y los programas de control, puede contribuir a la interpretación de la misma de manera comprensiva.

Aunque se han planteado en el pasado en otros contextos (21), este tipo de propuestas son escasas en la literatura y la historia de las zoonosis. Se espera avanzar en la comprensión e intervención de las zoonosis, al menos como se ha logrado en las políticas de ambiente y salud (22) y con respecto a la salud urbana (23).

¿Es una ventaja abordar el estudio de las zoonosis desde las ciencias de la complejidad? La respuesta no es simple ya que situar un problema en un contexto más amplio no aumenta por si sólo la comprensión del mismo.

\section{Complejidad}

La complejidad es reconocida como un paradigma alternativo, en construcción. En su significado más elemental, entendemos por paradigma un conjunto básico de creencias que guían la acción (24). Sin embargo, no se identifica aún como un patrón general de modelado teórico, desde las categorías que se utilizan hoy en día para etiquetar los paradigmas. 
Con denominaciones genéricas se reconocen los paradigmas positivista, pospositivista, las teorías críticas y el construccionismo; más recientemente se ha reconocido el paradigma participatorio (24). Estas denominaciones no restringen los campos disciplinarios, ya que, aunque hay disciplinas y corrientes de pensamiento que se ubican en un paradigma de manera exclusiva, son numerosas las disciplinas que agrupan corrientes positivistas, constructivistas y teorías críticas (por ejemplo, la sociología, la psicología, la economía, etc). A su vez, estas mismas disciplinas y otras similares albergan corrientes de pensamiento que hacen parte de las ciencias de la complejidad. Debe remarcarse que el conjunto de estos paradigmas es etiquetado desde la perspectiva de la complejidad como "ciencia clásica" (25), aunque probablemente el centro de la caracterización crítica corresponde al positivismo.

La identificación y diferenciación de los paradigmas se lleva a cabo por medio de sus bases ontológicas, epistemologícas y metodológicas. Así mismo, se utilizan temas básicos y críticos: Axiología (ética y valores); Acomodación y Comensurabilidad (¿Un paradigma cabe en otro?); Acción (qué hace el investigador); Control (¿Quién hace las preguntas?); Fundamentación de la verdad; Validez, y; Voz y representación.

El paradigma de la complejidad está construyendo sus bases ontológicas, epistemológicas y metodológicas; y cuenta con un acervo que le permite responder a gran parte de los temas básicos. Pero, es claro, que muchos temas son aún motivo de desarrollo o de controversia. Por tanto, no será posible describir el paradigma de la complejidad más que de manera aproximativa.

A continuación se presenta de manera sintética algunos elementos centrales de la complejidad, de orden ontológico (¿Cuál es la naturaleza de lo conocible, cual es la naturaleza de la realidad?), epistemológico (¿Cuál es la naturaleza de la relación entre el investigador y lo conocible?) y metodológico (¿Cómo el investigador puede conocer lo conocible?) (26-29).

En primer lugar debe mencionarse que la definición de complejidad varía según el área de conocimiento. Una de ellas plantea que se refiere a la "...pluralidad y jerarquización de niveles de ocurrencia de procesos, tanto en el sentido ontológico como en el sentido cognitivo...el término complejidad se puede referir a la diversidad de las relaciones entre los elementos componentes 
de un dado objeto modelo...la complejidad de un modelo puede ser entendida como su naturaleza no finalística o iterativa, correspondiendo en el lenguaje de la teoría de los sistemas a la propiedad de retroalimentación de modelos explicativos sistémicos." (26).

En esta perspectiva, desde el punto de vista ontológico, la complejidad asume que la realidad existe y es aprehensible, siempre y cuando se interprete como totalidad compleja, no determinista, reduccionista o lineal. Las visiones del mundo son traducciones y representaciones del mundo.

Desde el punto de vista epistemológico, el pensamiento complejo se precia de ser relativo, contextual, integral, así como apuntar a la unidad con multiplicidad y a la unidad con diversidad. Se reseñan a continuación, siete aspectos epistemológicos centrales de la complejidad, advirtiendo que no son los únicos:

- Lo dialógico: el orden y el desorden son dos lógicas contrapuestas y necesarias que pueden producir organización y complejidad. En la dialéctica de incertidumbre, complementariedad y de antagonismo se encuentra la complejidad.

- El principio recursivo rompe la idea lineal de causa efecto. Las relaciones determinantes son complejas, lo cual es clave cuando se considera la suma de las conductas de los componentes con respecto al comportamiento de la totalidad. La no linealidad es una propiedad de relaciones entre eventos que no siguen la lógica del efecto proporcional al estímulo causal (efecto dosis-respuesta), son discontinuos, iterativos, no convergentes ni finalísticos, y pueden tener efectos potencializadores de estímulos débiles en sistemas dinámicos complejos.

- El principio hologramático, según el cual la parte está en el todo y el todo está en la parte. Este principio conlleva autosimilitud e isomorfismo. Las cualidades y propiedades que nacen de la organización de un conjunto retroactúan sobre ese conjunto. Por otra parte, los problemas de organización social sólo pueden comprenderse a partir de este nivel complejo de la relación parte-todo. Así mismo, el conocimiento no se interrumpe: conocemos las partes, lo que nos permite conocer mejor el todo, pero el todo vuelve a permitir conocer mejor las partes.

- Relación entre el observador-conceptuador y el objeto observado, a diferencia del pensamiento simplificante que se basa en la disyunción absoluta entre el objeto y el sujeto que lo percibe y concibe. 
- El conocimiento se produce con base en condiciones bioantropológicas y socioculturales.

- La transdisciplinariedad, entendida como propiedad de objetos complejos $\mathrm{o}$ atributos de complejidad de relaciones entre campos disciplinarios. En estas relaciones, el principio de isomorfismo permite buscar entre clases de problemas aquellas que admiten una solución común, en lugar de buscar una solución para cada problema. Estas clases de universalidad sirven de base a la transdisciplinariedad (30). Sin embargo, para lograr una síntesis transdisciplinaria se requiere avanzar en síntesis paradigmáticas compartidas (26).

- Los fractales son figuras recurrentes cuya autosimilitud se manifiesta en el ámbito de escalas definidas. Se expresan como persistencia de formas, patrones y propiedades de los objetos en los diferentes niveles de su estructura jerárquica. Son producto de la iteración, repetición, de un proceso geométrico elemental que da lugar a una estructura final de una complicación aparente extraordinaria. Es decir que cada porción del objeto tiene la información necesaria para reproducirlo todo, y la dimensión fractal no necesariamente entera.

En lo metodológico, las ciencias de la complejidad utilizan estrategias, modelos y algoritmos para identificar complejidades de la realidad. Se encuentran aquellas que apuntan a identificar relaciones no lineales, isomorfismos, autosimilitud y fractalidad. Se cuenta con centenares de herramientas que abordan dimensiones específicas de la complejidad. La transdisciplinariedad es también un recurso metodológico cuando se le utiliza como proceso, estrategia de acción, modalidad de práctica, ya sea con un carácter instrumental o como práctica de transformación de disciplinas.

Sistemas complejos

La teoría de sistemas complejos hace parte de la complejidad. Por sistema complejo se entiende un sistema compuesto de múltiples partes interactuantes, o por la confluencia de múltiples procesos cuyas interrelaciones constituyen la estructura de un sistema que funciona como una totalidad organizada. De esta manera, el comportamiento colectivo del sistema es más que la suma de los comportamientos individuales. Esto se debe a que los vínculos y mutua dependencia de las partes crean información adicional y de las interacciones surgen propiedades nuevas que no pueden explicarse a partir de las propiedades de los elementos aislados. 
El concepto de "sistema complicado" es por completo diferente del presentado arriba. Un sistema complicado también puede estar compuesto de elementos heterogéneos y estos pueden multiplicar nexos de tipo administrativo, organizativo, de información, y similares, entre elementos de un mismo nivel jerárquico, pero no agregan valor al sistema total $(22,25,26)$.

La característica agregativa de un sistema complejo no equivale a capacidad sumativa. En otras palabras, excluye la posibilidad de obtener un análisis de un sistema complejo por la simple adición de estudios sectoriales correspondientes a cada uno de los elementos. Pero no excluye el papel de estudios parciales de alguno de sus elementos o de alguna de sus funciones. Por otra parte, la alteración en algunos elementos se propaga de diversas maneras a través del conjunto de relaciones que definen la estructura del sistema hasta, incluso, generar reorganización. Así mismo, las interacciones entre la totalidad y las partes no pueden ser analizadas fraccionando el sistema en áreas, según las disciplinas que estudian cada uno de los elementos, lo cual reedita la importancia de los enfoques interdisciplinarios y transdisciplinarios en el estudio de un sistema complejo. Pero una cosa son las interacciones y otra diferente un sistema como totalidad.

Para abordar el todo o las partes "... primero hay que especificar cómo se delimita una totalidad, cuál es la escala relativa y la jerarquía de sus partes relevantes, cuáles son los ejes en torno de los cuales se podrían establecer similitudes entre partes y conjuntos, y qué otros casos existen en los que se presenten correspondencias de la misma clase..." (30). En otros términos, para acercarse a la comprensión de un sistema complejo como totalidad es indispensable no sólo delimitarla, sino también identificar y caracterizar sus partes, componentes o elementos, y establecer sus relaciones e interacciones $(22,31)$, para lo cual se requiere abundante información. Esto genera enormes dificultades en la interpretación de los sistemas complejos, especialmente los de tipo social. A pesar de estas dificultades la teoría de sistemas complejos ha logrado significativos avances metodológicos en el análisis de la complejidad.

Como una primera conclusión se destaca que el reconocimiento de las zoonosis como problema complejo conduce a plantear un abordaje desde las ciencias de la complejidad, por dos razones centrales: Una primera, es claro 
que aún en el marco del reconocimiento de las zoonosis como problema complejo el abordaje analítico, político e intervencionista predominante se ha caracterizado por el reduccionismo. Esto, a pesar de su alcance formalista, no es de poca monta. En segundo lugar, porque las respuestas que se requieren para avanzar en las políticas y el control de las zoonosis deben desprenderse de enfoques que atiendan la causalidad estructural, el orden y desorden, la morfogénesis, la homeostasis, la teleología, entre otros, imposibles de abordar por medio de los métodos clásicos.

\section{Zoonosis en Colombia: sistema complejo y complejidad}

Se comentan unos campos de trabajo cuyo desarrollo permitan en el futuro enfocar o modelar las zoonosis desde la complejidad.

Un campo de trabajo es el relativo a la estructuración del sistema complejo de las zoonosis y su alcance en términos de complejidad.

Debe mencionarse que la delimitación de las zoonosis como totalidad no se opone al criterio reconocido de los límites borrosos que suelen presentar las totalidades sistémicas complejas. De hecho, la delimitación se acompaña de las escalas relativas en las que opera el sistema complejo como totalidad y el conjunto de sus partes en interacción. En el caso que nos ocupa podemos proponer cuatro tipos de escala, que incorporan los suprasistemas y los subsistemas: internacional, nacional, regional y municipal.

Dado que las interacciones de las partes constituyen la estructura y organización de un sistema complejo, se requiere identificar, de manera tentativa, el todo y las partes, con base en los conocimientos actuales que proporcionan diferentes disciplinas como la epidemiología, la economía, la sociología, la antropología, la historia y la salud pública, entre otras:

- Sistema total: zoonosis en Colombia.

- Suprasistema delámbito internacional (32-38): Demográfico; globalización económica, política y cultural; medio ambiente, incluido el cambio climático; políticas internacionales (ambientales, comercio y salud).

- Escala o sistemas del ámbito nacional: Modelo de desarrollo, tendencias demográficas, estructura económica y social, estructura y procesos políticos, modelos culturales, urbanización, medio ambiente (incluida la naturaleza y la vida silvestre) y seguridad alimentaria. 
- Escalas o subsistemas de los ámbitos regionales y municipales, que incluyen los mismos componentes del ámbito nacional, según la respectiva variedad y especificidad de las regiones y los municipios. Adicionalmente, son propios de estos ámbitos los sistemas productivos agropecuarios y agroindustriales, los hábitos y costumbres individuales y familiares y los sistemas y servicios de salud.

La tarea futura de identificar las jerarquías e interacciones entre los componentes de las diferentes escalas y entre las escalas, es una tarea de gran envergadura, así se lleve a cabo apelando únicamente a criterios de plausibilidad descriptiva y cualitativa. Esta tarea trasciende el alcance del presente ensayo. Sin embargo, cabe destacar algunos énfasis que resultan de los aportes de las disciplinas que se han ocupado de este tema:

1.Interacciones entre sistemas productivos, medio ambiente y poblaciones humanas y animales.

2.Interacciones entre la política, descentralización, servicios de salud, programas de control.

3.Interacciones entre las políticas públicas, sistemas productivos, medio ambiente y poblaciones

En este campo de trabajo también es un reto identificar las similitudes, autosimilitudes, isomorfismos, universalidades y problemas que admiten una solución común, en una perspectiva fractal de construcción del objeto zoonosis.

De manera tentativa, los ejes de lo biológico, social, político y cultural, en los cuales se dan los procesos de determinación de las zoonosis, pueden ser interpretados como parte de una dimensión fractal que atraviesa las escalas del sistema, ya comentadas. Esto podría dar lugar a una estructura fractal de red, con base en unidades fractales. A manera de ejemplo, es posible concebir una unidad fractal de organización social y económica: Sistemas productivos agropecuarios (producción agropecuaria moderna y tradicional; latifundios y pequeña propiedad campesina), población migrante y trabajadora; poblaciones humanas y animales domésticos y silvestres; hábitats y modos de circulación de agentes patógenos; programas de control.

Podría concebirse otra unidad de organización política e institucional: descentralización y conflicto armado; cultivos ilícitos y narcotráfico; 
movilidad poblacional; hábitats y modos de circulación de agentes patógenos; programas de control. Pero aún como ejemplos, en este caso es claro que se trata de una estructura fractal con bajo grado de autosimilitud.

Un segundo campo de trabajo debe generar abordajes interdisciplinarios y transdisciplinarios, aquellos que se requieren para la comprensión del sistema complejo y de la complejidad de las zoonosis.

Para avanzar hacia una síntesis interdisciplinaria y transdisciplinaria puede ser necesario generar convergencias entre los paradigmas que sirven de base a las disciplinas interesadas en las zoonosis (epidemiología, economía, sociología, antropología, etc.) (26) ya que trabajan desde sus propios horizontes teóricos y metodológicos y el conocimiento que aportan sobre un objeto individualizado y descontextualizado, se considera desestructurado. El conocimiento especializado de las disciplinas sobre los componentes del modelo complejo de las zoonosis genera la falsa idea de que estas partes son estables y su fragmentación es necesaria, ya que permite a cada elemento o componente explicarse a sí mismo. Esto dificulta estructurar el sistema complejo. Pero este conocimiento especializado es indispensable para encontrar algún grado de concordancia con respecto a los estatutos de cientificidad de los objetos en las respectivas disciplinas (26). Aproximaciones en ese sentido pueden lograse por medio de mapas de redes de relaciones entre disciplinas (http://wbpaley.com/images/ finalTopicMap5+1_.jpg).

Sobre esa base es posible buscar isomorfismos y clases de universalidad entre problemas que admitan soluciones en común, lo cual es significativo para replantear las zoonosis como un nuevo objeto complejo de estudio.

Un tercer campo de trabajo consiste en la identificación, aprehensión y prueba de múltiples herramientas para abordar la complejidad, ya que ninguna de ellas es efectiva en todos los escenarios. Algunas de las herramientas que en principio pueden ser compatibles con las características del sistema complejo y la complejidad de las zoonosis, planteadas arriba, podrían ser las siguientes (30):

- Los modelos de dinámica compleja (ecosistemas) y fractal y las simulaciones de comportamiento auto-organizado de multitudes (39). 
- Teorías de redes complejas, pequeños mundos, distribuciones independientes de escala y fractalidad (40).

- Geometría fractal en general y la dimensión fractal como medida de complejidad, ya que algunas estructuras sociales exhiben organizaciones fractales (41).

- Modelos de agentes autónomos, vida, sociedades y culturas artificiales: usan inducción para encontrar patrones en los datos, o deducciones para hallar consecuencias emanadas de los supuestos. Han constituido el territorio de ciencias emergentes: econofísica, biofísica, geofísica, etc. (42).

- Teorías de transiciones de fase con sus principios de clases de universalidad, renormalización y escalamiento: a escala adecuada existen unas pocas clases de problemas formalmente idénticos cualesquiera sea el dominio empírico (43).

Si bien debe reconocerse que lo anteriormente planteado es sólo una aproximación a la comprensión de las zoonosis como problema complejo de salud pública, es conveniente superar los enfoques explicativos lineales y unidisciplinarios (44-46) dominantes por muchos años y acercarse a las ciencias de la complejidad. Afortunadamente existe evidencia en la actualidad que justifica abordar las zoonosis desde la complejidad y desde ópticas inter y transdisciplinarias $(14,26,45-47)$.

Para responder de la manera más apropiada a los retos que las enfermedades zoonóticas nos presentan, es necesario atender tres aspectos de orden teórico y metodológico: retomar las zoonosis como un problema complejo, desarrollar enfoques interdisciplinarios y transdisciplinarios $(9,48)$ y, abordar el problema desde la complejidad. De esta manera, se aportará al desarrollo de programas de control apropiados, prestando especial atención a las zoonosis que provienen de la vida silvestre $(8,49)$

\section{REFERENCIAS}

1. Calvo M ¿Nuevas Zoonosis del siglo XXI. Real Academia de Ciencia Veterinarias de Madrid. Madrid; 2005.

2. Cárdenas J. Situación en Colombia y Latinoamérica de las zoonosis. mvz-cordoba. 2000; 5(1):41-5.

3. Mahy B, Dykewicz C, Fisher-Hoch S, Ostroff S, Tipple M, Sanchez A. Virus zoonoses and their potential for contamination of cell cultures. Dev. Biol. Stand. 1991: 75:183-89.

4. Organización Mundial de la Salud Estadísticas Sanitarias Mundiales. 2012. Ginebra: Organización Mundial de la Salud; 2012. 
5. OPS/OMS. Propuesta del plan estratégico 2008-2012. Documento oficial 328; 2007.

6. Villamil LC, Romero JR, Soler D. Salud pública veterinaria, bienestar de la humanidad. Bogotá: Universidad de la Salle; 2012.

7. Jones K, Patel N, Levy M, Storeygard A, Balk D, Gittleman J, et al. Global trends in emerging infectious diseases. Nature. 2008; 451:990-994.

8. Chomel B, Belotto A, Meslin F. Wildlife, exotic pets, and emerging zoonoses. Emerging Infectious Diseases. 2007; 13(1):6-11.

9. Nishi J, Gragon D, Elkin B, Mitchell J, Ellsworth Tr, Hugh-Jones M. Emergency response planning for anthrax outbreaks in bison herds of northern Canada: a balance between policy and science. Resources, Wildlife, and Economic Development (RWED), Government of the Northwest Territories (GNWT), Fort Smith, Northwest Territories. Canada; 2006.

10. Organización Mundial de la Salud. Subsanar las desigualdades en una generación. Informe final, Comisión sobre Determinantes Sociales de la Salud. Argentina; 2009.

11. Schramm FR, Castiel LD. Processo Saúde/Doença e Complexidade em Epidemiologia. Cad. Saúde Públ. Rio de Janeiro. 1992; 8 (4): 379-390.

12. Morgan M, Spicer J, Reid M. Sociological and psychological investigations. In: Detels R, McEwen, Beaglehole R, Tanaka E. (Ed.). Oxford Textbook o Public Health. Fourth Edition. Vol. 2. New York: Oxford University Press; 2002. pp. 781.

13. Camacho B. Revisión de la política de zoonosis en Colombia a partir de mediados del siglo XX. Bogotá: Maestría en Salud Pública; 2010.

14. Medlock J, Jameson L. Ecological approaches to informing public-health policy and risk assessments on emerging vector-borne zoonoses. Emergin Health Threats Journal. 2010; 3(1): 58-67.

15. Agudelo P. Leptospirosis: Diagnóstico serológico. Rev CES Med. 2005; 19(1):37-41.

16. Ferro E, Rodríguez L, Pérez M, Travi B. Seroprevalencia de infección por Leptospira en habitantes de barrios periféricos de Cali. Biomédica. 2006; 26:250-257.

17. Góngora A, Parra J, Aponte L, Gómez L. Seroprevalencia de Leptospira spp. en grupos de población de Villavicencio, Colombia. Rev. Salud pública (Bogotá). 2008; 10(2):269278.

18. Rodríguez G. Estado actual de la leptospirosis. MVZCórdoba. 2000; 5(1):61-63.

19. Herrera J, Vergara C, Vivas C, Falconar A. Comportamiento de la leptospirosis en el departamento del Atlántico (Colombia). SaludUninorte. 2005; 20:18-29.

20. Mesa F, Cárdenas J, Villamil L. Las encefalitis equinas en la salud pública. Primera edición. Universidad Nacional de Colombia, Facultad de Medicina Veterinaria y de Zootecnia; 2005.

21. Marques M. Doenças Infecciosas Emergentes no Reino da Complexidade: Implicações Para as Políticas Científicas e Tecnológicas. Cad. Saúde Públ. 1995: 11(3):361-78.

22. Rolando G. Interdisciplinariedad y sistemas complejos. Revista Latinoamericana de Metodología de la Ciencias Sociales. 2011; 1(1):66-101.

23. International Council for Science. Health and Wellbeing in the Changing Urban Environment: a Systems Analysis Approach. Paris: ICSU; 2011.

24. Lincoln YA, Lynham SA, Guba EG. Paradigmatic controversies, contradictions, and emerging confluences, revisited. In: Denzin HK, Lincoln YS. Handbook of qualitative research. USA: SAGE Publications, Inc.; 2011. p. 97.

25. Morin E. La epistemología de la complejidad. Gazeta de Antropología. 2004 (20) Articulo 02 (http://hdl.handle.net/10481/7253).

26. Almeida N. Complejidad y transdisciplinariedad en el campo de la salud colectiva: Evaluación de conceptos y aplicaciones. Salud Colectiva. 2006: 2(2):123-46.

27. Morin E. On the Definition of Complexity. En: Aida E, editor. The Science and Praxis of Complexity. Tokyo: United Nations University; 1984. p.62-68.

28. Morin E. Introduction à la Pensée Complèxe. París: Editions Sociales Françaises; 1990.

29. Samaja J. Epistemología de la Salud. Buenos Aires: Lugar Editorial; 2004. 
30. Reynoso C. Modelos o metáforas. Crítica del paradigma de la complejidad de Edgar Morin. Buenos Aires: Editorial Sb; 2009.

31. Shalizi CR. Methods and techniques of complex systems science: An overview. Center for the Study of Complex Systems. USA: University of Michigan; 2003.

32. Louria DB. Emerging and re-emerging infections: the societal variables. Int J Inf Dis. 1996;1:59-62.

33. Satcher D. Emerging infections: getting ahead of the curve. Emerg Infect Dis. 1995; 1:1-6.

34. Piñon $A$, Barceló $C$. Situacion ambiental y su relación con afecciones a la salud. Rev Cubana Med Gen Integr. 2005: 21:3-4.

35. Romero $\mathrm{H}$, Vasques A. Evaluación ambiental del proceso de urbanización en las cuencas del piedemonte andino de Santiago de Chile. Rev eure. 2005: 31(94):97-118.

36. Gil A, Samartino L. Zoonosis en los sistemas de producción animal de las áreas urbanas y periurbanas de América Latina. Food and Agriculture Organization; 2000.

37. Rivera O. La bioseguridad y la zoonosis ante el cambio climático. 2do Congreso Panamericano de Zoonosis. Bogotá-Colombia; 2009.

38. Varela N. Enfermedades Relacionadas con la Tenencia y Manejo de Fauna Silvestre. Grupo de Estudio de Animales Silvestres. Boletín GEAS. 2002: 4(1):4-13.

39. Brunk $\mathrm{G}$. Why do societies collapse?: A theory based on self-organized criticality. Journal of Theoretical Politics. 2002; 14 (2): 195-230.

40. Barabási A. Linked: How everything is connected to everything else and wath it means. Nueva York: Plume Books; 2003.

41. Losa G, Merlini D, Nonnenmacher T, Weibel E (Compiladores). Fractals in biology and medicine. Vol. 4. Basilea-Boston-Berlin: Birkhäuser Verkag; 2005.

42. Agar M. Agents in living colors: Towards emic agent-based model. Journal of Artificial Societies and Social Simulation. 2005; 8 (1): 4 ( http://jasss.soc.surrey.ac.uk/8/1/4. $\mathrm{html}$ ).

43. Hirsh H, Hoffmann-Riem H, Biber-Klemm S, Grossenbacher-Mansuy W, Joye D, Christian $D$, et al. (Compiladores). Handbook of transdisciplinary research. Dordrecht: Springer; 2008.

44. Arch E, Rosado J. Ciencias de la complejidad y caos como herramientas en el análisis de la proliferación de vectores y zoonosis. Cirugía y cirujanos. 2009: 77(24):341-50.

45. Coker R, Rushton J, Mounier-Jack S, Karimuribo E, Lutumba P, Kambarage D, et al. Towards a conceptual framework to support one-health research for policy on emerging zoonoses. The Lancet Infectious Diseases. 2011; 11(4):326-31.

46. Matamoros J, Sanín L, Santillana M. Las Zoonosis y sus Determinantes Sociales: Una Perspectiva a Considerar en Salud Pública. Rev. Salud Pública (Bogotá). 2000; 2 (1): 17-35.

47. Cutler S, Fooks A, Van Der Poel. Public health threat or new, reemerging and neglected zoonoses in the industrialized world. Emerging Infectious Diseases. 2010; 16 (1):1-7.

48. Keusch G, Pappaioanou M, González M, Scott K, Tsai P. Sustaining Global Surveillance and Response to Emerging Zoonotic Diseases. The National Academies of Sciences; 2009.

49. Mills J, Gage K, Khan A. Potential influence of climate change on vector-borne and zoonotic diseases: A review and proposed research plan. Environmental Health Perspectives. 2010;118(11):1507-1514. 